\title{
Stationary Axisymmetric Solutions of the Einstein Equations with Rigidly Rotating Perfect Fluid and Nonlinear Charged Sources *
}

\author{
Humberto Salazar \\ FCFM, Benemérita Universidad Autónoma de Puebla, \\ A.P. 1152, 72000 Puebla, México. \\ Rubén Cordero \\ Departamento de Física, CINVESTAV-IPN, \\ A.P. 14-740, 07000 México, D.F., México.
}

\begin{abstract}
A class of stationary rigidly rotating perfect fluid coupled with nonlinear electromagnetic fields was investigated. An exact solution of the Einstein equations with sources for the Carter $\mathrm{B}(+)$ branch was found, for the equation of state $3 p+\epsilon=$ constant. We use a structural function for the Born-Infeld non-linear electrodynamics which is invariant under duality rotations and a metric possessing a fourparameter group of motions. The solution is of Petrov type $\mathrm{D}$ and the eigenvectors of the electromagnetic field are aligned to the DebeverPenrose vectors.
\end{abstract}

PACS: 04.20.Jb; 04.40.+c

*Supported in part by CONACyT. 


\section{BORN-INFELD NON-LINEAR ELECTRODYNAMICS AND DUALITY ROTA- TIONS}

The basic description of the dynamical equations of non-linear electrodynamics within general relativity can be done in terms of the null tetrad formalism according to which the metric is given by

$$
g=2 e^{1} \otimes e^{2}+2 e^{3} \otimes e^{4}, \quad e^{2}=\overline{e^{1}}
$$

where the $e^{a} \in \Lambda^{1}$ fulfill the Cartan structure equations

$$
d e^{a}=e^{b} \wedge \Gamma_{b}^{a}=\Gamma_{b c}^{a} e^{b} \wedge e^{c}
$$

and $\Gamma_{b}^{a} \in \Lambda^{1}$ satisfy the second structure equations

$$
d \Gamma_{b}^{a}+\Gamma_{s}^{a} \wedge \Gamma_{b}^{s}=\frac{1}{2} R_{b c d}^{a} e^{c} \wedge e^{d}
$$

The Riemann curvature components $R_{b c d}^{a}$ may be replaced by the Weyl conformal tensor components, which are characterized by five complex curvature coefficients $C^{(a)}$, and the components of the traceless Ricci tensor $C_{a b}=R_{a b}-1 / 4 g_{a b} R$ where $R_{a b}=R_{a b s}^{s}$ and $R=R_{a}^{a}$. Non-linear theories of Born Infeld type (B-I) are theories with a hamiltonian function $\mathcal{H}$ depending on the invariants of the skew-symmetric tensor $P_{a b}$,

$$
\begin{gathered}
P=\frac{1}{4} P^{a b} P_{a b} \quad Q=\frac{1}{4} \check{P}^{a b} P_{a b} \\
\check{P}^{a b}=-\frac{1}{2} \epsilon^{a b c d} P_{c d}
\end{gathered}
$$

where $\epsilon^{a b c d}$ is the Levi-Civita symbol with $\epsilon^{1234}=1$. 
Skew-symmetric tensor $F_{a b}$ can be defined by the material equations

$$
\begin{gathered}
F_{a b}=\mathcal{H}_{P} P_{a b}+\mathcal{H}_{Q} \check{P}_{a b} \\
\mathcal{H}_{P}=\frac{\partial \mathcal{H}}{\partial P} \quad \mathcal{H}_{Q}=\frac{\partial \mathcal{H}}{\partial Q}
\end{gathered}
$$

We select the null tetrad in such a manner that out of all independent components of the electromagnetic field tensors $F_{a b}$ (corresponding to $\vec{E}$ and $\vec{B}$ ) and $P_{a b}$ (corresponding to $\vec{D}$ and $\vec{H}$ ), they are different from zero only:

$$
P_{34}=D, \quad P_{12}=i H, \quad F_{34}=E, \quad F_{12}=i B
$$

where $D, H, E$, and $B$ are real. The above selection for $F_{a b}$ and $P_{a b}$ can be made simultaneously by virtue of the material equations. The invariants of $F_{a b}$ and $P_{a b}$ read:

$$
\begin{aligned}
\frac{1}{4} P^{a b} P_{a b}+\frac{1}{4} \check{P}^{a b} P_{a b} & =P+Q=-\frac{1}{2}(D+i H)^{2} \neq 0 \\
\frac{1}{4} f^{a b} f_{a b}+\frac{1}{4} \check{f}^{a b} f_{a b} & =F+G=-\frac{1}{2}(E+i B)^{2} \neq 0
\end{aligned}
$$

So that $(D, H)$ and $(E, B)$ can be interpreted as independent parameters of the complex invariant of the electromagnetic field. We now can introduce by a Legendre transformation of the Hamiltonian $\mathcal{H}(\mathrm{P}, \mathrm{Q})$ or $\mathcal{H}(\mathrm{D}, \mathrm{H})$ a new structural function for the non-linear electrodynamics given by (Salazar et al., 1987)

$$
M(D, B)=B H-\mathcal{H}(D, H)
$$

In the following we are going to use the same notation that Salazar et al. (1987) 
Furthermore we restrict our structural function $M$ to be invariant under duality rotations i.e.

$$
M\left(D^{\prime}, B^{\prime}\right)=M(D, B) \quad \text { for } \quad D^{\prime}+i B^{\prime}=e^{i s}(D+i B)
$$

The last condition can be easily seen to constrain the function $M$ to be a function of the variable $\left(D^{2}+B^{2}\right)$ only:

$$
M=b^{2} f(X), \quad X \equiv \frac{1}{2 b^{2}}\left(D^{2}+B^{2}\right), \quad b=\text { constant } .
$$

The original Born-Infeld theory given by the Hamiltonian function

$$
\mathcal{H}=b^{2}-\sqrt{b^{4}-2 b^{2} P+Q^{2}}
$$

belongs to this class of theories invariant under duality rotations and corresponds to

$$
f(X)=\sqrt{1+2 X}-1
$$

\section{EINSTEIN BORN INFELD EQUATIONS WITH PERFECT FLUIDS}

In this work we are concerned with solutions to the Einstein-Born-Infeld equations with a perfect fluid

$$
\begin{aligned}
R_{a b}-\frac{1}{2} g_{a b} R & =-T_{a b} \\
T_{a b} & =(p+\epsilon) u_{a} u_{b}+p g_{a b}-8 \pi E_{a b} \\
u_{a} u^{a} & =-1
\end{aligned}
$$




$$
\begin{aligned}
p+\epsilon & >0 \\
4 \pi E_{a b} & =\mathcal{H}_{p}\left(-P_{a s} P_{b}^{s}+g_{a b} P\right)+\left(P \mathcal{H}_{P}+Q \mathcal{H}_{Q}-\mathcal{H}\right) g_{a b} \\
\check{F}_{a b ;}{ }^{a} & =0 \\
P^{b a ;}{ }_{a} & =4 \pi J^{b}
\end{aligned}
$$

where $E_{a b}$ is the energy-momentum tensor of the non-linear electromagnetic field, $u_{a}$ is the fluid four-velocity, $p$ is the fluid pressure and $\epsilon$ is the energy density. We shall consider the Carter type D metric with conformal factor and having a four-parameter group of symmetries. We shall work with the standard gravitational units so chosen that the gravitational constant $G$ and the velocity of light $c$ are equal to the unity. Let now $\varepsilon$ be a dimensionless constant, $l$ be a constant of dimension of length, and $x^{\mu}:=\xi, \bar{\xi}, r, \tau$ and $x^{\mu}:=u, v, r, \sigma$ be two coordinate charts: $\xi$ is complex, while the remaining coordinates and constants are real. The $r, \tau$ and $\sigma$ are of dimension of length while $\xi, u$ and $v$ are dimensionless. Given these ingredients, we construct first the two 2-dimensional Riemannian spaces of constant curvature

$$
\Lambda^{1} \otimes \Lambda^{1} \ni d l^{2( \pm)}:=\left\{\begin{array}{l}
4 \frac{d \xi \otimes d \bar{\xi}}{(1+\varepsilon \xi \bar{\xi})^{2}} \\
4 \frac{d u \otimes d v}{(1+\varepsilon u v)^{2}}
\end{array}\right.
$$

given in terms of stereographic coordinates $(\xi, \bar{\xi})$ and the associated real one-forms of dimension of length

$$
\Lambda^{1} \ni \pi^{ \pm}:=\left\{\begin{array}{l}
d \tau+2 i l\left(\frac{\bar{\xi} d \xi-\xi d \bar{\xi}}{1+\varepsilon \xi \bar{\xi}}\right) \\
d \sigma+2 l\left(\frac{v d u-u d v}{1+\varepsilon \xi \bar{\xi}}\right)
\end{array}\right.
$$

Then, introducing two real analytic functions $N=N(r)$ and $F^{( \pm)}=$ $F^{( \pm)}(r)$ (are of dimension (lenght) $)^{2}$, we define now the 4-dimensional Riemannian space-time of signature $(+++-)$ 


$$
B^{( \pm)}: d s^{2}:=N d l^{2( \pm)}+\frac{N}{F^{( \pm)}} d r \otimes d r \mp \frac{F^{( \pm)}}{N} \pi^{( \pm)} \otimes \pi^{( \pm)}
$$

It can be easily shown that the (Carter, 1968) separable $B^{( \pm)}$branches of type D can be brought — without any loss of generality — to the form of (13). In the considered representation of the $B^{( \pm)}$metrics, only the sign of the parameter $\varepsilon$ is relevant.

We should like now to explain why we consider (13) as the optimal coordinatization of the Carter $B^{( \pm)}$branches for our purpose. We observe first that a formal transformation

$$
z \rightarrow-u, \quad \bar{z} \rightarrow-v, \quad \tau \rightarrow i \sigma, \quad F^{(+)} \rightarrow F^{(-)}
$$

which obviously implies $d l^{2(+)} \rightarrow d l^{2(-)}, \pi^{(+)} \rightarrow i \pi^{(-)}$brings the $B^{(+)}$metric into the $B^{(-)}$metric. We will see that this leads to a useful computational advantage; the natural tetrads; connection and curvatures of $B^{( \pm)}$metrics can be treated via perfectly parallel computations. Secondly, we notice that our coordinatization of the $B^{( \pm)}$metrics allows us to give a unified description of their minimal sub-group of symmetries, which are 4-dimensional Lie Groups. Next, taking the $B^{(+)}$metrics, we should like to investigate the advantages of our coordinatization from the point of view of the Debney-Kerr-Schild formalism described in the first section. With the metrics (13) a natural choice for the null tetrads is correspondingly

$$
\begin{gathered}
-\frac{\sqrt{2 N}}{1+\varepsilon \xi \bar{\xi}}\left\{\begin{array}{l}
d \xi \\
d \bar{\xi}
\end{array}=\left\{\begin{array}{l}
e^{1} \\
e^{2}
\end{array}\right.\right. \\
\frac{1}{\sqrt{2}}\left[\left(\sqrt{\frac{N}{F}}\right) d r \pm \sqrt{\frac{F}{N}} \pi\right]=\left\{\begin{array}{l}
e^{3} \\
e^{4}
\end{array}\right.
\end{gathered}
$$


whereas the connection forms are given by

$$
\begin{aligned}
\Gamma_{42} & =\sqrt{\frac{F}{2 N^{3}}}(i l-1 / 2 \dot{N}) e^{1} \\
\Gamma_{31} & =\sqrt{\frac{F}{2 N^{3}}}(i l-1 / 2 \dot{N}) e^{2}
\end{aligned}
$$

where dots denote the $\mathrm{r}$ derivative

$$
\Gamma_{12}+\Gamma_{34}=\frac{\varepsilon}{\sqrt{2 N^{3}}}\left[\xi e^{2}-\bar{\xi} e^{1}\right]-\frac{1}{\sqrt{2}}\left[\left(\sqrt{\frac{F}{N}}\right)+i l \sqrt{\frac{F}{N^{3}}}\right]\left(e^{3}-e^{4}\right)
$$

From the second structure equations

$$
\begin{gathered}
d \Gamma_{42}+\Gamma_{42} \wedge\left(\Gamma_{12}+\Gamma_{34}\right)=\gamma e^{3} \wedge e^{1}+\delta e^{4} \wedge e^{1} \\
d \Gamma_{31}+\left(\Gamma_{12}+\Gamma_{34}\right) \wedge \Gamma_{31}=\delta e^{3} \wedge e^{2}+\gamma e^{4} \wedge e^{2} \\
d\left(\Gamma_{12}+\Gamma_{34}\right)+2 \Gamma_{42} \wedge \Gamma_{31}=\beta e^{1} \wedge e^{2}+\alpha e^{3} \wedge e^{4}
\end{gathered}
$$

where

$$
\begin{gathered}
\alpha=\left[1 / 2\left(\frac{F}{N}\right)+i l\left(\frac{F}{N^{2}}\right)\right] \\
\beta=\frac{F}{N^{3}}(i l-1 / 2 \dot{N})^{2}-\frac{\varepsilon}{N}+2 i l \sqrt{\frac{F}{N^{3}}}\left[\left(\sqrt{\frac{F}{N}}\right)+i l \sqrt{\frac{F}{N^{3}}}\right] \\
\gamma=1 / 2 \frac{F^{1 / 2}}{N}\left[\frac{2}{N^{1 / 2}}(i l-1 / 2 \dot{N})\left(\left(\sqrt{\frac{F}{N}}\right)+i l \sqrt{\frac{F}{N^{3}}}\right)-\frac{F^{1 / 2}}{N}\left(i l \frac{\dot{N}}{N}+\ddot{N}+1 / 2 \frac{\dot{N}^{2}}{N}\right)\right]
\end{gathered}
$$




$$
\delta=-\frac{F}{8 N \dot{N}}\left(\frac{4 l^{2}+\dot{N}^{2}}{N}\right)
$$

we can obtain the Weyl coefficients $C^{(a)}$, the traceless Ricci tensor $C_{a b}$ and the scalar curvature $R$

$$
\begin{gathered}
C^{(3)}=\frac{1}{3}(\alpha+\beta+2 \gamma) \\
R=2(\alpha+\beta-4 \gamma) \\
C_{12}=\frac{1}{2}(\beta-\alpha)=-C_{34} \\
C_{33}=-2 \delta=C_{44} .
\end{gathered}
$$

Other curvature components are equal to zero.

We shall consider rigidly rotating perfect fluid in the comoving frame such that the four-velocity in tetrad and coordinate components reads

$$
\begin{gathered}
u_{1}=0=u_{2} ; \quad u_{3}=-u_{4}=1 / \sqrt{2} \\
u^{\mu}=\sqrt{\frac{N}{F}} \delta_{\tau}^{\mu}
\end{gathered}
$$

Then the Einstein equations coupled with B-I theories invariant under duality rotations and perfect fluid become (Salazar et al., 1987)

$$
\begin{gathered}
R=-8 b^{2} f(X)+8 b^{2} X f \nabla(X)+3 p-\epsilon \\
C_{12}=-2 b^{2} X f \nabla(X)-1 / 4(p+\epsilon)
\end{gathered}
$$




$$
C_{33}=-1 / 2(p+\epsilon)
$$

denoting the derivative of $f(X)$ with respect to $X$ by superscript $\nabla$.

From the definition

$$
J^{\mu}=\rho u^{\mu}
$$

we can infer that $X$ depends only on $r$

$$
X=X(r)
$$

and the Born-Infield equations read

$$
[N(B+i D)]^{j}-2 i l f \nabla(X)(B+i D)=4 \pi i N J^{\tau}
$$

Solving Eqs. (31)-(36) we arrive to the equations for the pressure $p$ and the energy density $\epsilon$

$$
\begin{gathered}
p=2 b^{2} f(X)-\frac{\varepsilon}{N}+\frac{\dot{F} \dot{N}}{2 N^{2}}-\frac{F}{4 N^{3}}\left(4 l^{2}+\dot{N}^{2}\right) \\
\epsilon=-2 b^{2} f(X)+\frac{\varepsilon}{N}-\frac{\dot{F} \dot{N}}{2 N^{2}}+\frac{3 F}{4 N^{3}}\left(4 l^{2}+\dot{N}^{2}\right)-\frac{F \ddot{N}}{N^{2}} \\
p+\epsilon=-\frac{F}{2 N \dot{N}}\left(\frac{4 l^{2}+\dot{N}^{2}}{N}\right) \neq 0
\end{gathered}
$$

and for the electromagnetic field

$$
\begin{gathered}
B=\frac{a_{0}-2 l A}{N} b \quad D=\frac{\dot{A} b}{f \nabla(X)} \quad A=A(r) \\
a_{0}, b=\text { constants }
\end{gathered}
$$




$$
f \nabla(X)=\dot{A} / \sqrt{2 X-\left(\frac{a_{0}-2 l A}{N}\right)^{2}} \quad X=X(A)
$$

the remaining Einstein equation is

$$
-2 b^{2} X f \nabla(X)=\left[-2 \varepsilon-\ddot{F}+\frac{2 \dot{F} \dot{N}}{N}-\frac{F\left(4 l^{2}+\dot{N}^{2}\right)}{N^{2}}\right] / 4 N
$$

For every set of functions $A, F, N$ satisfying Eq. (42) we have an analytic solution of the Einstein-Born-Infeld equations coupled with a perfect fluid. Energy density and pressure are given by (37), (38) and the electromagnetic field is given by (40).

\section{SOME EXPLICIT EXAMPLES:}

\section{A. Solution without electromagnetic field}

When we switch off the electromagnetic field $X=0, A=a_{0} / 2 l$ we recover from Eq. (42) the equation given by Kramer et al. (1987)

$$
-2 \varepsilon-\ddot{F}+2 \frac{\dot{F} \dot{N}}{N}-F \frac{\left(4 l^{2}+\dot{N}^{2}\right)}{N^{2}}=0
$$

B. Linear electromagnetic field

When

$$
f(X)=X
$$


Eq.(42) reduces to

$$
(\dot{A})^{2}+\frac{\left(a_{0}-2 l A\right)^{2}}{N^{2}}+\frac{1}{4 N}\left[-2 \varepsilon-\ddot{F}+2 \frac{\dot{F} \dot{N}}{N}-F \frac{\left(4 l^{2}+\dot{N}^{2}\right)}{N^{2}}\right]=0
$$

this solution was reported by García and Tellez (1992).

C. Linear electromagnetic field coupled with dust

When we couple to the gravitational and linear electromagnetic field dust, characterized by the condition $p=0$, then

$$
F=\varepsilon r^{2}+C_{1} r+C_{2} \quad C_{1}, C_{2}=\text { constants }
$$

and Eq. (42) reduces to

$$
\dot{A}^{2}+\frac{\left(a_{0}-2 l A\right)^{2}}{N^{2}}-\frac{1}{2 N} \dot{F} \dot{N}+\frac{F}{4 N^{3}}\left(4 l^{2}+\dot{N}^{2}\right)+\frac{\varepsilon}{N}=0
$$

this solution is not reported in the literature.

\section{Born-Infeld original theory}

For the original B-I theory described by $f(X)=\sqrt{(1+2 X)}-1$, Eq. (42) reduces to

$$
\begin{gathered}
b^{2} \frac{(\dot{A})^{2}+\left(a_{0}-2 l A\right)^{2} / N^{2}}{\sqrt{\left(1-(\dot{A})^{2}\right)\left[1+\left(a_{0}-2 l A\right)^{2} / N^{2}\right]}}+ \\
\frac{1}{4 N}\left[-2 \varepsilon-\ddot{F}+\frac{2 \dot{F} \dot{N}}{N}-\frac{F\left(4 l^{2}+(\dot{N})^{2}\right)}{N^{2}}\right]=0
\end{gathered}
$$


this branch of solutions has been not reported in the literature, except for $A=c t e \neq \frac{a_{0}}{2 l}$ (Bretón, 1989).

\section{E. Born-Infeld theory with perfect fluid and given equation of state}

From Eqs. (37) and (38) we arrive to the general equation

$$
(3 p+\epsilon)^{\dot{2}}=4 b^{2} f^{\nabla}(X) \frac{\left(X N^{2}\right)}{N^{2}}-\frac{F}{N^{2}}\left[\ddot{N}-\frac{4 l^{2}+(\dot{N})^{2}}{N}\right]
$$

with the ansätz

$$
X=\frac{\varepsilon_{0}^{2}+g_{0}^{2}}{N^{2}} \quad \varepsilon_{0}, g_{0}=\text { constants }
$$

and the equation of state

$$
3 p+\epsilon=c t e=\gamma_{0}
$$

Eq. (49) reduces to

$$
\left[\ddot{N}-\frac{4 l^{2}+\dot{N}^{2}}{N}\right]=0
$$

with general solution

$$
\begin{gathered}
N=\beta_{0}+\sqrt{\frac{4 l^{2}}{\beta_{1}}+\left(\beta_{0}\right)^{2}} \cosh \sqrt{\beta_{1}}\left(r-\beta_{2}\right) \\
\beta_{0}, \beta_{1}, \beta_{2}=\text { constants }
\end{gathered}
$$

then from the equation of state we obtain for $F$

$$
F=\dot{N}\left[F_{0}+\int^{r} \frac{N}{(\dot{N})^{2}}\left[\left(\gamma_{0}-4 b^{2} f\left(N^{-2}\right)\right) N+2 \varepsilon\right] d y\right]
$$

on the other hand, from

$$
X=\frac{\varepsilon_{0}^{2}+g_{0}^{2}}{N^{2}}
$$


we have two possibilities for the electromagnetic field $B+i D$ :

i) Magnetic solution

$$
\begin{gathered}
A=\text { constant } \neq \frac{a_{0}}{2 l} \\
B=\frac{g_{0}}{N} \\
D=0 \\
4 \pi J^{\tau}=\frac{2 l g_{0}}{N^{2}} f \nabla\left(\frac{g_{0}^{2}}{N^{2}}\right)
\end{gathered}
$$

ii) Generalized NUT solution

$$
\begin{gathered}
\text { for } \quad A \neq c t e \\
B+i D=\frac{2 b^{2}\left(\varepsilon_{0}^{2}+g_{0}^{2}\right)}{N} e^{i \phi} \\
\phi=2 l \int^{r} \frac{1}{N} f^{\nabla}\left(\frac{1}{N^{2}}\right) d y \\
J^{\tau}=0
\end{gathered}
$$

Case ii) generalizes the NUT type D solution of the Einstein-Born-Infeld equations (Salazar et al, 1987) when we add a rigidly rotating perfect fluid.

\section{CONCLUSIONS}

We have gotten a wide set of solutions of the Einstein equations coupled with rigidly rotating perfect fluid and linear and non-linear electromagnetic field.

One of them, given by Eq. (57), generalizes the NUT solution in the case of Born-Infeld and perfect fluid sources satisfying the equation of state $3 p+\epsilon=$ constant. 


\section{REFERENCES}

Bretón B. N. (1989). Journal of Mathematical Physics, 30, 2607.

Carter B. (1968). Commun. Math. Phys. 10, 280.

García, D.A. and Tellez, J. (1992). Journal of Mathematical Physics, 33, 2254 .

Kramer et al. (1980). Exact Solutions of Einstein's Field Equations, Cambridge University Press.

Salazar, H. I., García, D.A., and Plebanski, J.F. (1987). Journal of Mathematical Physics 28, 2171. 